\title{
Correlation Filters for Automatic Fire Detection Systems
}

\author{
HEINZ O. LUCK \\ Duisburg University \\ Electrical and Electronic Engineering \\ Dept. of Communications \\ Bismarckstr. 81, D-4100 Duisburg, FRG
}

\section{ABSTRACT}

The technical development in automatic fire detection is mainly governed by the rapidly increasing development of electronic components including microprocessors that opens a variety of new signal processing tools. Most applications used in automatic fire detection systems are aimed at the improvement for system handling in the alarm or in the fault signal situation or at the improvement of automatic system monitoring. Only rather few attempts have been made to use modern electronic tools including software solutions to improve the detection capability. This article deals with a method to develop signal detection algorithms which can be used in automatic fire detection systems and which, in addition, can very well be realized as software controlled electronic circuits, e.g. as software program operated microcomputers or as special VLSI circuits. The method is based on a simple and fairly general model for the physically measurable signal in the vicinity of a developing fire and is therefore not restricted to a special kind of fire detector.

Keywords:

Automatic fire detection, detection capability, fire detection algorithm, correlation filters.

\section{INTRODUCTION}

Any extinguishing measure in a developing fire situation requires a preceeding detection procedure. From this point of view fire detection is a most important part in the chain of fire prevention measures and far too little attention has been payed to the associated techniques in the past compared to other important fields. The situation can be very roughly characterized as follows:

- Only rather few characteristic fire parameters physically measurable in the vicinity of a developing fire are used for detection purposes. These parameters are mainly temperature rise, smoke concentration and flame radiation intensity in the IR- and UV-wavelength range $/ 1 /$.

- As far as the detection procedure itself is concerned rather poor signal processing methods have been applied in the past. The alarm signal mainly is created within the detector head by a simple threshold comparison. Signal and data transmission require no sophisticated techniques. Only few detectors are grouped together to protect small areas in the building so that an alarm from a detector group instantaneously indicates the location of the developing fire in the building in a very simple manner.

- The important electrical connection lines within fire detection systems e.g. between detectors and the control and indicating equipment or to 
remote control stations (fire-brigade) are monitored automatically and fault signals are indicated differently from the alarm signals on the indicating panel.

In some special cases a more sophisticated signal and data processing procedure was required and therefore it has been introduced. If the false alarm problem became too incomfortable some coincidence techniques between two different detector groups or a suitable time delay for the alarm signal generation with the check whether or not the alarm criterion holds within the delay time were introduced. Furthermore it was necessary to provide flame detectors with more intelligent signal processing tools in order to bring them into operation at all.

This short review indicates that the classical fire detection technique does not require very challenging signal detection tools. The advantages associated to this classical detection technique are:

- Its performance is easy to understand.

- It is inexpensive (e.g. low developing costs are involved).

- It results in robust systems and installations.

- The reliability is quite satisfactory and the lifetime for components and installations can fairly well be estimated.

- The efficiency has obviously been satisfactory too (the main figure is in the range of one false alarm per year and 100 detector heads).

But there are some severe shortcomings which are the main reason for a rather bad image associated with this technique.

- The associated efficiency cannot be increased any more. For large installations with several hundred detector heads or large areas with large numbers of installations that have to be supervised by one fire brigade the small figure of "one false alarm per year and 100 detectors" is no longer satisfactory. In addition it is a matter of fact that the absolute number of false alarms considerably exceeds the number of real fire alarms $12 /$.

- Automatic monitoring and control for correct performance is mainly restricted to the power supply components and some electrical signal lines.

- The actual state of the important system components is not automatically monitored. Partial control of the system is mainly provided by some majntenance procedures which are performed in some regular time intervals.

The application of microprocessor techniques introduces the possibility to realize important system properties by software solutions in a very flexible manner. Microprocessors and software solutions in the development of fire detection systems are aimed at the following goals:

(a.) Improvement and facilitation for system handling in the case of alarm or in fault signal situations, where some measures have to be taken.

(b.) Improvement of automatic system monitoring which may result in a higher reliability level with the consequence that the maintenance requirements be reduced.

(c.) Improvement of the detection capability.

In all the three areas considerable progress is possible if a suitable use is made of new signal and data processing facilities. Many attempts have been made already concerning the first two points $15,6 /$. But rather few work concerning the improvement of detectivity is known to the author so far 13, 4/. If the term detection capability is defined as the capability of a fire detection system to safely distinguish between the situations "Fire" and "Not Fire" it is obviously the essential parameter in this context at all. Therefore this article deals with item (c.) in the following.

\section{THE BACKGROUND FOR SIGNAL DETECTION FILTERS IN AUTOMATIC FIRE DETECTTON SYSTEMS}

Fig. 1 shows the basic procedure in an automatic fire detection installation. The following paper only deals with the signal processing part $m(t) \rightarrow y(k)$ and 


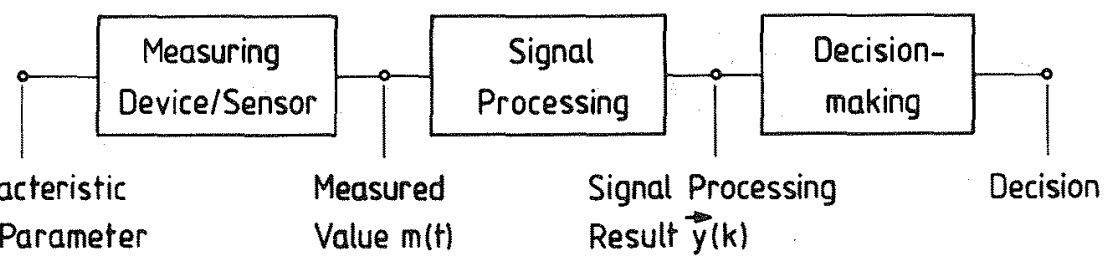

Fig. 1 Signal Processing in Autmatic Fire Detectors

does not consider the type of sensor which may be used. The most simple mathematical model for $m(t)$ is given by

$m(t)= \begin{cases}s_{j}(t)+n(t) & \text { if a fire signal is present } \\ n(t) & \text { in normal conditions }\end{cases}$

$\left\{s_{j}(t)\right\}$ is the set of different signal components in $m(t)$ which may be produced by ${ }^{j}$ different fire conditions. $n(t)$ represents the measured value without any fire influence.

In automatic fire detection systems an extremely high efficiency and reliability is required. Compared with a "normal" signal detection situation the following aspects are of mayor importance in this context:

(1.) The signal detection has to be as fast as possible because the associated costfunction depends very much on the detection time $/ 7 /$.

(2.) The false alarm rate must be extremely low because any countermeasure initiated in an alarm situation may cause unexceptable costs.

(3.) The signals $\{s,(t)\}$ to be detected are not known in all their details because they are determined by a very large number of different parameters.

(4.) There is only few information available about the noise $n(t)$ that has to be taken into account.

In this situation the classical detection filter theory based on the matched filter concept is no longer applicable. Some other techniques, e.g. nonparametric signal detection, has to be used. In the following the methods to be used are discussed more systematically.

- All signal components are considered as signal vectors with a finite number n of components:

$m(t) \rightarrow \vec{m}=\left[\begin{array}{c}m_{1} \\ m_{i} \\ m_{n}\end{array}\right]$ and $s(t) \rightarrow \vec{s}_{j}=\left[\begin{array}{c}s_{1} \\ s_{j i} \\ s_{n}\end{array}\right]$ and $n(t) \rightarrow \vec{n}=\left[\begin{array}{c}n_{1} \\ \dot{n}_{j} \\ \dot{n}_{n}\end{array}\right]$

$\vec{m}= \begin{cases}\vec{s}_{j}+\vec{n}_{\vec{n}} & \text { if a fire is present } \\ & \text { under normal conditions }\end{cases}$

The limited number of signal vector components can be derived by a suitable sampling procedure if an observation interval of limited duration $T_{0}$ is considered.

It is not known in advance which of the possjble signals $s_{j}(t)$ will occur in a real fire case. Therefore it is necessary to match the detection algorithm to those properties of the signals $s .(t)$, that are common to all elements of the set of possible fire signals $\left\{\vec{j}_{s_{j}}(t)\right\}$. So the component $\vec{s} j$ is considered as an element $\vec{s}$ of a set of signals $\{\vec{s}\}$ where all the elements in the set have some common properties which are significant for this set of signals. This may be shown by a simple example. 
Fig. 2 shows some typical signals $s_{j}(t)$ measured in a fire experiment where the noise $n(t)$ is suppressed. There are two different smoke density measurements taken simultaneously and both serve as signals to be detected in the same dangerous situation. They are different but they have some common properties which are significant for the situation. It can easily be seen that in all possible observation periods of length $\mathrm{T}_{0}$ both signals have a positive or "increasing" trend.

This property can be indicated by using a ranking technique where each measured signal vector

$\vec{s}_{j}=\left[\begin{array}{c}s_{j 1} \\ s_{j i} \\ s_{j n}\end{array}\right] \Rightarrow \vec{r}_{s j}=\left[\begin{array}{l}r_{s j 1} \\ \dot{r}_{s j i} \\ \dot{r}_{s j n}\end{array}\right]$

is associated with a rank vector $\vec{r}$

The components $r$ are the ranksj of the associated signal components $s$, within the signal vector $\vec{s}$ according to their magnitude. Using this notation every type of trend within the signal vector easily can be indicated. In particular all monotonously increasing signals have the same rank vector

$\vec{r}_{\text {nat }}=\left[\begin{array}{c}1 \\ \dot{i} \\ \dot{n}\end{array}\right]$

A positive trend can now be defined by having the rank vector $\vec{r}$ in some neighbourhood of $\vec{r}$. In other words: In this example the set ${ }^{s}$ f signals $\{s\}$ to be detected can be defined by the significant and common signal property of a positive trend in the observation period $\mathrm{T}$.

As a general result we are looking for detection filters for the detection of predetermined signal properties which are associated with a set of otherwise different signals.

\section{GENERALIZED MATCHED FILTER CONCEPT /8/}

In order to get a mathematical (or calculable) formulation it is necessary to have suitable definitions for

- the general term "signal properties" and

- a quality measure associated with a criterion of optimization.

Definitions:

For convenience the subscript $j$ in $\left\{s_{j}\right\}_{\text {will }}$ be $\operatorname{dropped}$ so that $\vec{s}_{j}=\vec{s}$.
(1.) The modified sign function:

$$
u(\xi)=\left\{\begin{array}{r}
+1 \text { for } \xi>0 \\
0 \text { for } \xi \leq 0
\end{array}\right.
$$

(2.) The term "signal property" is defined by any transformation of $\overrightarrow{\mathrm{m}}$ or $\vec{s}$ resp. of the form:

$$
\vec{f}_{i}(\vec{m})=\left[\begin{array}{c}
f_{i 1}(\vec{m}) \\
\dot{f}_{i j}(\vec{m}) \\
\dot{E}_{i n}(\vec{m})
\end{array}\right] \text { or } \quad \vec{g}_{i}(\vec{s})=\left[\begin{array}{l}
g_{i 1}(\vec{s}) \\
\dot{g}_{i j}(\vec{s}) \\
\dot{g}_{i n}(\vec{s})
\end{array}\right]
$$

where $\overrightarrow{\mathrm{f}}_{i}$ and $\overrightarrow{\mathrm{g}}_{j}$ in general are vector functions of $\overrightarrow{\mathrm{m}}$ or $\overrightarrow{\mathrm{s}}$. We briefly discuss an example, which afterwards will be used: 


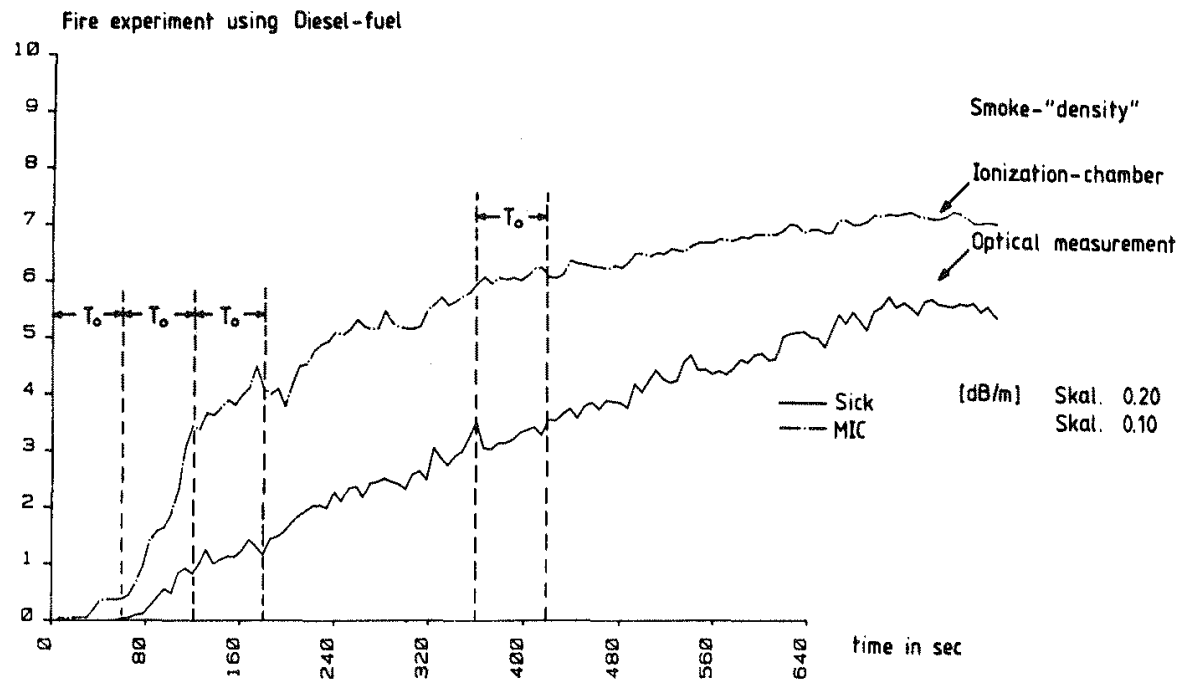

Fig. 2 Typical Fire Signals

Example:

The "signal property" is the order of a single signal sample si within the signal vector $\vec{s}$ according to the signal sample magnitudes and can be indicated by

$\vec{g}_{i}(\vec{s})=\left[\begin{array}{l}u\left(s_{1}-s_{i}\right) \\ u\left(s_{j}-s_{i}\right) \\ u\left(s_{n}-s_{i}\right)\end{array}\right]$

Other "signal properties" do not require a notation of such complexity. In these cases the vector function $\overrightarrow{\vec{f}}$ or $\vec{g}$, resp. are reduced to simple scalar values. The conclusion which can be drawn from this kind of definition is that every transformation or function of $\vec{s}$ that can be written in the form of a vector $\vec{f}_{i}(\vec{s})$ can serve as mathematical model for a signal property.

$A^{i}$ convenient "quality measure" is a generalized correlation coefficjent introduced by Kendall /9/ which can be written in the following form ( $\mathrm{f}^{\mathrm{T}}$ indicates the transposed matrix):

$c=\frac{\sum_{i=1}^{n} \vec{f}_{i}^{T}(\vec{m}) \vec{g}_{i}(\vec{s})}{\sqrt{\sum_{i=1}^{n} \vec{f}_{i}^{T}(\vec{m}) \overrightarrow{+}_{i}(\vec{m})} \sqrt{\sum_{i=1}^{n} \vec{g}_{i}(\vec{s}) \vec{g}_{i}(\vec{s})}}$

Using the Cauchy-Schwartz inequality we have

$\sum_{i=1}^{n} \vec{f}_{i}^{T}(\vec{m}) \vec{g}_{i}(\vec{s}) \leqslant \sqrt{\sum_{i=1}^{n} \vec{f}_{i}^{T} \vec{f}_{i}} \sqrt{\sum_{i=1}^{n} \vec{g}_{i} \vec{g}_{i}}=\sqrt{E_{m}} \sqrt{E_{s}}$ 
and $c \leqslant 1$. The terms

$E_{m}=\sum_{i=1}^{n} \vec{f}_{i}^{T}(\vec{m}) \vec{f}_{i}(\vec{m})$ and $E_{s}=\sum_{i=1}^{n} \vec{g}_{i}^{T}(\vec{s}) \vec{g}_{i}(\vec{s})$

may be considered as generalized signal "energies".

The coefficient $C$ serves as a similarity measure between the property $\overrightarrow{\mathrm{f}}(\overrightarrow{\mathrm{m}})$ of the filter input $\vec{m}$ and the property $\vec{g}(\vec{s})$ of the signal $\vec{s}$. We now regard $\vec{g}(\vec{s})$ as that signal property which is to be detected or, in other words, $\{\vec{s}\}^{i}$ is regarded as a predetermined set of signals which is defined by the known signal property $\vec{g}_{i}(\vec{s})$. So $\vec{g}_{i}(\vec{s})$ and $E_{s}$ become constants and we have:

$\frac{1}{\sqrt{E_{m}}} \sum_{i=1}^{n} \vec{f}_{i}(\vec{m}) \vec{g}_{i}(\vec{s}) \leqslant \sqrt{E_{s}}$

Obviously the left hand part of this relation approaches its maximum value $\sqrt{E_{S}}$ if

$\vec{f}_{i}(\vec{m})=\vec{g}_{i}(\vec{s})$ for all $i$

Following this line the detection filter can be based on Kendall's correlation coefficient choosing $\overrightarrow{\mathrm{f}}_{i}=\vec{g}_{i}$

$\left.C_{\mathrm{T}}=\frac{1}{\sqrt{E_{\mathrm{m}}}} \sum_{i=1}^{n} \overrightarrow{\mathrm{g}} \overrightarrow{\mathrm{T}}_{\mathrm{i}} \overrightarrow{\mathrm{m}} \overrightarrow{\mathrm{g}} \overrightarrow{\mathrm{i}}_{\mathrm{i}} \overrightarrow{\mathrm{s}}\right) \leqslant \sqrt{\mathrm{E}_{\mathrm{s}}}$

Where $C_{\mathrm{r}}$ serves as a quality measure and the above relation is the associated criterion of optimization, i.e. $C_{T}$ has to be made as large as possible at most equal to $\sqrt{\mathrm{E}}$.

In the abseñce of noise, $i . e \cdot \vec{m}=\vec{s}$ the filter output produces its maximum value if the filter input $\overrightarrow{\mathrm{m}}$ belongs to the set $\{\vec{s}\}$ of signals defined by $\vec{g}$ ( $\vec{s}$ ). From this point of view we have a detection filter "matched" to all signals $\vec{s}$ belonging to $\{\vec{s}\}$ or, in other word, "matched" to all signals $\vec{s}$ with the signal property $\vec{g}_{i}(\vec{s})$. Such a detector may be called a generalized matched filter. Necessarily the special form of $\vec{g}_{1}(\ldots)$ has to be known.

The filter behaviour in a noisy background cannot be treated in such a general way. Furthermore it is beyond the scope of this article. In the following some examples shall be discussed.

\section{Example 1:}

If the signal property to be detected is the signal trend it can be based on a suitable indication of the individual ranks for all signal components according to theix numerical values.

$$
\vec{g}_{i}(\vec{s})=\left[\begin{array}{c}
u\left(s_{1}-s_{i}\right) \\
\dot{u}\left(s_{j}-s_{i}\right) \\
\dot{u}\left(s_{n}-s_{i}\right)
\end{array}\right] \text { and } \vec{g}_{i}(\vec{m})=\left[\begin{array}{l}
u\left(m_{1}-m_{i}\right) \\
\dot{u}\left(m_{j}-m_{i}\right) \\
\dot{u}\left(m_{n}-m_{i}\right)
\end{array}\right]
$$

and Kendall's generalized correlation coefficient (3.3) yields

$$
y\left(T_{0}\right)=C_{T}=\frac{1}{\sqrt{E_{m}}} \sum_{i=1}^{n} \sum_{i=1}^{n} u\left(m_{j}-m_{i}\right) u\left(s_{j}-s_{i}\right) \leqslant \sqrt{E_{s}}
$$


with

$E_{m}=\sum_{i=1}^{n} \sum_{j=1}^{n} u\left(m_{j}-m_{i}\right), \quad E_{s}=\sum_{i=1}^{n} \sum_{j=1}^{n} u\left(s_{j}-s_{i}\right)$

Consider now the property of a monotonously increasing signal $\vec{s}$ which has a strictly increasing trend and

$u\left(s_{j}-s_{i}\right)=u(j-i)=\left\{\begin{array}{lll}1 & \text { for } j>i \\ 0 & \text { for } j \leqslant i\end{array}\right.$

so that

$E_{s}=\sum_{i=1}^{n} \sum_{j=1}^{n} u(j-i)=\frac{n}{2}(n-1) ; E_{m} \leqslant \frac{n}{2}(n-1)$

$y\left(T_{0}\right)=\sum_{i=1}^{n} \sum_{j=1}^{n} u\left(m_{j}-m_{i}\right) \leqslant \frac{n}{2}(n-1)$

This is the well known (nonlinear) nonparametric Kendall-t-detector for trend. It has a considerable advantage because it can be rewritten in a recursive form

$y(k)=y(k-1)-\sum_{i=1}^{n} u[m(k-(n-i))-m(k-n)]+\sum_{i=1}^{n} u[m(k)-m(k-(n-i))]$

An associated filter structure can very easily be derived as is shown in Fig. 3 .

\section{Example 2:}

Many applications in fire detection yield the following signal property: For all signals $s(t)$ it is possible to define a generalized (time dependent) threshold function $m_{0}(t)$ within the observation period $T_{0}$ with

$s(t)>m_{0}(t)$ for all $t \varepsilon\left[0, T_{0}\right]$

as can be seen from Fig. 2 .

If we introduce a suitable threshold $m_{0}(t)$ as a standard signal

$m_{0}(t) \rightarrow \vec{m}_{0}=\left[\begin{array}{l}m_{01} \\ \dot{m}_{0 i} \\ \dot{m}_{o n}\end{array}\right]$

and consider the difference $\vec{s}-\vec{m}_{0}>0$ i.e. $s_{i}>m_{0 i}$, for all $i$, as that signal property to be detected, we get

$\vec{g}_{i}(\vec{s})=g_{i}(\vec{s})=g_{i}\left(s_{i}\right)=u\left(s_{i}-m_{O i}\right)=1$ $\left.\vec{g}_{i}(\vec{m})=g_{i}(\vec{m})=g_{i}\left(m_{i}\right)=u\left(m_{i}-m_{o i}\right) \quad\right\}$

and 


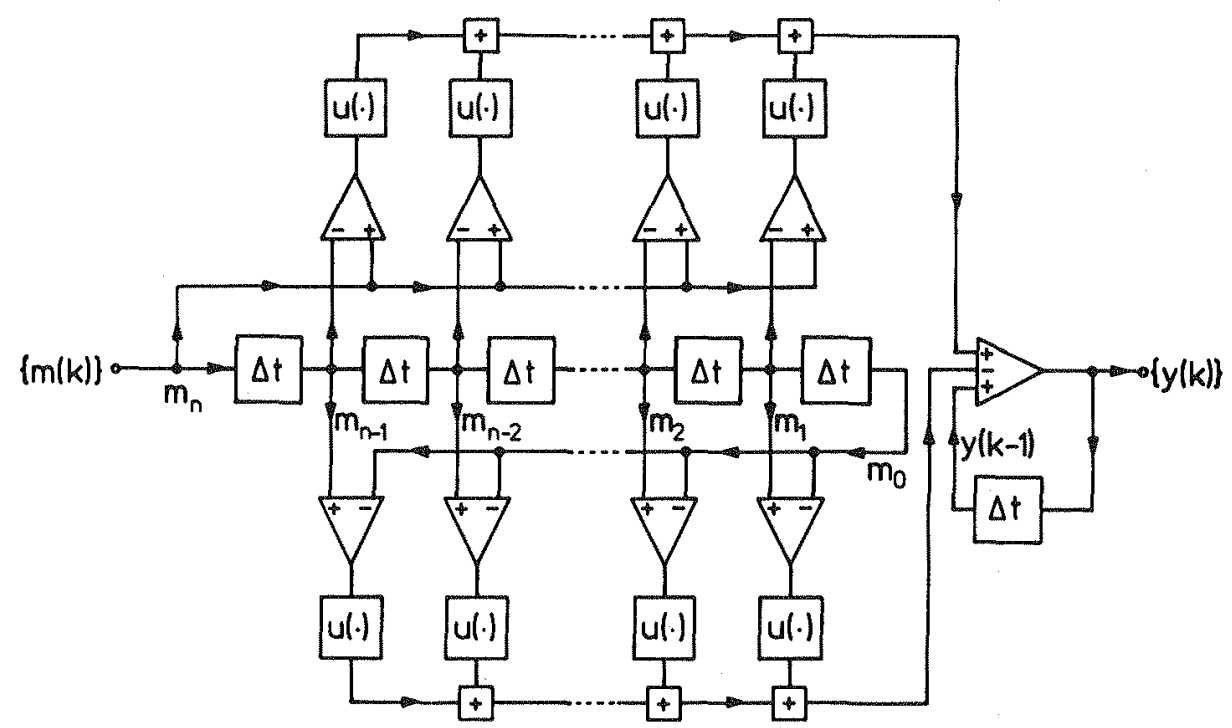

Fig. 3 Trend Detection Filter (Kendall-t Type)

$E_{s}=\sum_{i=1}^{n} u\left(s_{i}-m_{o i}\right)=n ; E_{m}=\sum_{i=1}^{n} u\left(m_{i}-m_{o i}\right) \leqslant n$

$y\left(T_{0}\right)=C_{T}=\sum_{i=1}^{n} u\left(m_{i}-m_{o i}\right) \leqslant n$ or written as a recursive algorithm

$\left.y(k)=\sum_{i=1}^{n} u\left[m(k-(n-i))-m_{o i}\right)\right] \leqslant n$

Example 3:

In some important fire detection cases the signals to be detected very rapidly rise to a rather high signal energy. A simple signal energy detector can be derived by using the method indicated above. This results in

$y\left(T_{0}\right)=C_{T}=\sum_{i=1}^{n} m_{i}^{2} \leqslant n$

or written in a recursive form

$y(k)=\sum_{i=1}^{n} m^{2}(k-(n-i))$

which is a normalized signal energy detector.

\section{APPLICATIONS}

In practical applications mostly a combination of several different signal properties define a set of signals $\{\vec{s}\}$ which are to be detected, or in other 
words, the detection filter has to be matched to a set of signal properties. Following the theory outlined above a fire detector is a combination of several single detection filters each of them matched to a special property. A logic connection has then to be made according to the detection situation which is to be solved. Fig. 4 shows an automatic fire detection system based on the signal properties outlined above.

A prefiltering shown in Fig. 4 is to reduce the influence of highly correlated noise and has not been discussed in this presentation $/ 10 /$.

\section{FIRE DETECTION CAPABILITY}

Up to this point the interference by the noise component has been neglected. It has not been incorporated in the method of the detection filter design. On the other hand it has a considerable influence upon the efficiency of the fire detector performance.

The filter behaviour in a noisy background cannot be treated in such a general way mainly because no general information about the noise is available. Nevertheless there is some evidence from field experiments for the assumption that the noise $n(t)$ consists of at least two different components

$n(t)=n_{0}(t)+n_{t}(t)$

where $n_{0}(t)$ is a low pass approximative stationary signal so that the associated sample-vector $\vec{n}$ contains statistically dependent components. $n_{t}(t)$ is a rather rare and transient signal which is very hard to measure in the field.

Under this condition it is hardly possible to calculate the detector performance efficiency in terms of false alarm or detection probability or any other statistical measure.

The only way to overcome this problem is to use some simulation technique.

In order to study the behaviour and the efficiency of detector types discussed in this article a special simulation and measuring device has been used $/ 4 /$. It is computer-controlled and generates the input data with suitable accuracy and simulates the detector algorithm. In addition, the important and interesting statistical parameters of both input data as well as detector output can be measured and plotted in a suitable form. The basic configuration of the device is discussed elsewhere $16 /$. In this context the following items are of major importance.

(1) It is important to use a simulation method which generates reliable results much faster than they can be drawn from measurements in the field. This is mainly because false alarms are very rare events in practical cases if one single detector device or even a group of several detectors is considered. On the other hand, it is obviously impossible to draw any reliable conclusion from a statistical measurement without having observed at least $10^{3}$ false alarms.

(2) The main difficulty in this context is the fact that a signal generator is needed to produce a variety of simulated input signals for the detector device, which allows to determine both the distribution function or probability density $p_{m}(m)$ of the generated signal $\{m\}$ and its autocorrelation function $R_{m m}(k)$ independently from each other. $R_{m m}(k)$ is a measure for the two-dimensional dependency of the signal samples $\mathrm{m}(\mathrm{k})$ and is therefore closely. linked to the two-dimensional probability density function. So a practical solution cannot comply with the above requirement in principle. On the other hand, there is - within some mathematical limits - an approximate solution which cannot be outlined in this article. Some details are given in $/ 4 /$.

Results from several simulation experiments using the method indicated above show that fire detection systems based on detection filters shown in Fig. 4 very well may be able to improve the fire detection capability, i.e. reliabi- 


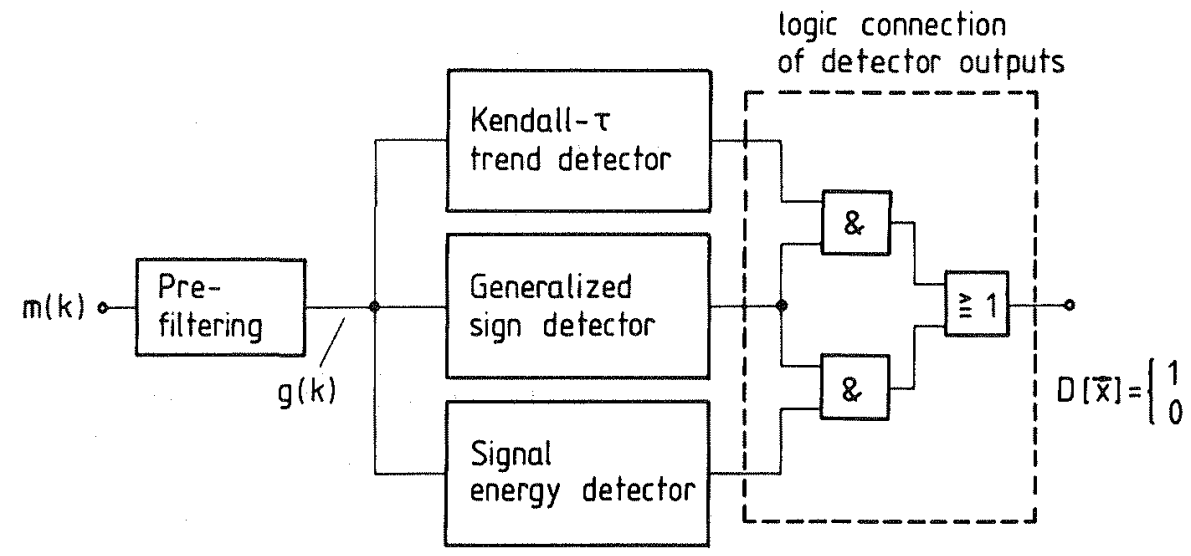

Fig. 4 Fire Detector Matched to a Combination of Signal and Noise Properties

lity of detection as well as false alarm rate, to a considerable amount. Algorithms like those mentioned above can easily be implemented by using modern microcomputer means as software programs or they can be introduced as specific VLSI-circuits both without an unsuitable increase in system prices. Future development will, probably, increasingly be directed towards an improm vement of fire detection capability using modern electronic means including software solutions. This, on the other hand, is necessarily going to change the testing methods and associated international standards.

\section{REFERENCES}

$11 / \mathrm{H}$. Luck, "Neuere Entwicklungen und Entwicklungstrends in der Technik der automatischen Brandentdeckung", Tagungsbericht Fachtagung des VBSF und des BVD, Nov. 1983, Bern, Schweiz

121 J.F. Fry, "The Problem of False Alarms from Fire Detection systems", Proceedings of 6 th International Seminar on Probleme der automatischen Brandentdeckung', Institut für Elektrische Nachrichtentechnik, RWTH Aachen, Oct. 1971, pp. 37-53

13/ R. v. Tomkewitsch, "Fire Detector Systems with 'Distributed Intelligence' - The Pulse Polling System", Fire Safety Journal 6 No. 3 (1983)

14/ H. Luck and K.-R. Hase, "Signal Detection Aspects in Automatic Fire Detection", Fire Safety Journal 6 No. 3 (1983)

15/ Fire Safety Journal 6 No. 3 (1983), (Special Issue on Automatic Fire Detection)

16/ H. Luck (ed.), "Proceedings of AUBE 182 - 8. Internationales Vortragsseminar über Probleme der automatischen Brandentdeckung", Universität Duisburg, Oktober 1982

17/ H. Luck, "Wirksamkeitsmaße für spezielie Signaldetektoren einfacher Struktur", AEÜ 26 (1972) pp. 267-275.

18/ H. Luck, "Signal Detection Using a General Matched Filter Concept", AEÜ 36 (1982) pp. 217-222

$19 /$ M. Kendal1, "Rank Correlation Methods", Griffin, 4th edn. London 1975

$110 / \mathrm{H}$. Luck, "Signal Detection in Automatic Security Systems", Proceedings of EUSIPCO-83 (2nd European Conference on Signal Processing), H.W. Schüssler (ed.), Elsevier Science Publishers B.V. (North-Holland)(1983). 